\title{
Influence of exercise order on the number of repetitions in untrained teenagers.
}

Eveline Moraes', Marcelo Pontes Nobre², Marianna de Freitas Maia ${ }^{3}$, Belmiro de Freitas Salles ${ }^{4}$, Humberto Miranda ${ }^{5}$, Roberto Simão ${ }^{6}$.

\begin{abstract}
Introduction: Prescription of resistance training (RT) is increasing among teenagers, requiring further studies to investigate the effects on this population. Objective: To examine the different orders on exercises sequence and its influence on the number of repetitions in teenager. Methods: Participated in the study 12 voluntarily male teenagers (14.91 \pm 0.79 years, $1.69 \pm 8.61 \mathrm{~cm}, 62.24 \pm 7.26 \mathrm{~kg}$ ) without experience in RT. After 48 hours of the last session of 10 repetitions maximum (10RM) test, subjects performed one of the four sequences of exercises in a counterbalanced crossover design. Four different sequences were also performed with a interval of 48 hours. All sequences were three sets of each exercise with 10RM load, with two-minute intervals between sets and exercises. The order of exercises was the sequence 1: bench press (BP), pulldown (PD), machine shoulder press (SP), biceps curl (BC) and triceps (TR); Sequence 2: TR, BC, SP, PD and BP; the sequence 3: Leg press (LP), leg extension (LE) and leg curl (LC); Sequence 4: LC, LE and LP. Results: Significant differences were observed $(p<0.05)$ in the total number of repetitions for the BP exercises, SP, BC and TR for the sequence of the upper limbs and for the lower limbs and the LP and LE exercises. Conclusion: The results suggest that the performance of the average number of repetitions for sessions conducted with RT intensity of 10RM in teenagers was influenced by the different kind of exercises in both upper and lower limbs.
\end{abstract}

Keywords: Resistance training; Muscle performance; Exercise order.

\section{INTRODUCTION}

Resistance training (RT) is a safe and effective method of conditioning for children and teenagers when performed in a safe environment with the correct exercise technique and proper supervision. ${ }^{(1)}$ The participation of children and teenagers in a RT program can improve not only the strength and the muscular resistance, (2) but also body composition, lipid profile, bone mineral density, (3) cardiovascular conditioning and mental health. ${ }^{(4,5)}$ All these factors can reduce the risk of injuries in sports and recreational activities. ${ }^{(1)}$

The National Strength Conditioning Association (NSCA) ${ }^{(1)}$ recognized and supported, in 2009, the premise that many of the benefits associated with RT programs targeted for adults are attainable by children and adolescents who follow specific guidelines according to age. ${ }^{(1)}$ In their position about RT for children and adolescents, the NSCA ${ }^{(1)}$ discussed on the variable order of the exercises, but can be seen that there is not a primary reference basing such claims.

The American College of Sports Medicine (ACSM) ${ }^{(6)}$ suggested in 2009 that, for the best performance of the exercises, they should be sequenced from the largest to the smallest grouping, from multi-joint exercises to the single-joint exercises and from higher intensity exercises to the lower intensity exercises. ${ }^{(6)}$ Other recommendations include alternate the exercises for lower and upper limbs or agonist/antagonist manner, i.e., conducting an exercise to a muscle and then another exercise to the opposite grouping. Is noteworthy that the studies ${ }^{(7-10)}$ cited by ACSM (7) are directed to adults.

Simão et al. ${ }^{(11)}$ in a literature review examined the order of the exercises and their influence in acute and chronic responses. The review shows that regardless of the amount of muscle mass involved in the exercise, the number of

Corresponding Author: Marianna de Freitas Maia Address: Rua General Sezefredo, 310 apto. 407 Cep: 21715-062- Rio de Janeiro - RJ Telephone (21) 998458144 E-mail: mariannamaia2@gmail.com

${ }^{3}$ Professora Mestre Substituta, Escola de Educação Física e Desportos da Universidade Federal do Rio de Janeiro - UFRJ, Rio de Janeiro (RJ), Brasil. 
repetitions is higher when the exercise is positioned at the beginning of a training program. In terms of chronic adaptations related to the increase of strength and hypertrophy, greater increases in strength and hypertrophy were evident in individuals not trained in the exercises that initiated the training sequences. Thus, the authors suggested that exercises must be sorted on based on priority of importance according to the purpose of the training program regardless of the exercise involves a large or small muscle group. After this review of Simão et al. ${ }^{(11)}$ other studies have been published, but self-respecting their differences in the materials and methods, suggested that regardless of the ordering of exercises of the individual in a RT session should start by the prioritized muscle group. ${ }^{(12-14)}$

Regarding the ordination of exercises and teenagers, the first publication of our knowledge is by Romano et al., (15) who investigated the acute effects of different exercises orders on the number of repetitions performed in teenagers. Thirteen male adolescents performed two sequences for upper limbs. The authors concluded that the number of repetitions is distinguished significantly in different orders and major exercises should be performed first.

Considering the importance of exercises order in the prescription of RT and the absence of studies on the subject for children and teenagers, information on the effects of different exercise orders in healthy untrained adolescents seems justified. Thus, the aim of this study was to examine the different orderings of a sequence of exercises for upper limbs (UL) and lower limbs (LL) and its influence on the number of repetitions in teenagers. Our hypothesis, based on the evidence for adults, is that the exercises order will affect the performance of the number of repetitions, regardless of exercise and muscle mass involved.

\section{METHOD}

\section{Sample}

The sample consisted of 12 male adolescents (14.91 \pm 0.79 anos; $1.69 \pm 8.61 \mathrm{~cm} ; 62.24 \pm 7.26 \mathrm{~kg}$ ) without previous experience with RT. The inclusion criteria were: a) do not perform any other type of regular physical activity during the study, beyond what was prescribed; b) not present functional limitations to the performance of the RT program or test; c) not make use of nutritional supplements before or during the study; d) be classified between stages 3 and 4 on the Tanner scale, through self-assessment, because the method of self-assessment of the maturational stage is considered a reliable and reproducible alternative. ${ }^{(16)}$ Subjects were instructed to not participate in another RT program. The experimental procedures were performed in accordance with the Declaration of Helsinki and the Ethics Committee of the Santa Casa de Misericordia Institution from Juiz de Fora approved the study protocol $(009 / 12)$.

\section{Determination of the loads of 10 maximum repetitions (10MR)}

Four load assessments were performed in 10MR. In these assessments were included testing and retesting of $10 \mathrm{MR}$ separated by 48-72 hours for the same muscle group. Individuals were distributed in the test by the Latin square method and the order was repeated in the re-test. To minimize possible errors in the $10 \mathrm{MR}$ tests, the following strategies were used: ${ }^{(11)}$ (a) all participants received standard instructions on the general routine of evaluation data and exercise performance techniques before the data collection; (b) the exercises execution technique was monitored and corrected if necessary; (c) all subjects received verbal encouragement during testing. Before the tests, a general warming up exercise was performed in the cycle ergometer with five minutes duration followed by a specific heating performed with light-load for 15 repetitions on the first exercise of the session. During the $10 \mathrm{MR}$ tests, each participant performed a maximum of five attempts in each exercise with three to five minutes break between them. After the $10 \mathrm{MR}$ load in a specific exercise was determined, a 10-minute interval was allowed before starting the $10 \mathrm{MR}$ test of the following exercise. There was no pause between the eccentric and concentric phases of the repetitions.

\section{Experimental protocols}

Forty-eight hours after the last 10MR test session subjects performed one of the four sequences of exercises in a counterbalanced crossover design. The four different sequences were performed with a 48-hour interval. Before each sequence of exercises, was conducted a warm-up consisted of five minutes in the cycle ergometer followed by 12 repetitions on the first session of the exercise with $40 \%$ of $10 M R$ load. Two minutes after warming up, subjects performed the exercise sequence. All sequences were composed of three sets for each exercise with load to 10MR and intervals of two minutes between sets and exercises. The exercise order was Sequence 1: Bench Press (BP), Front Pull-down (PD), Machine Shoulder Press (SP), Biceps curl (BC) and Triceps (TR); Sequence 2: TR, BC, SP, PD and BP; Sequence 3: Leg press (LP), Leg Extension (LE) e Leg Curl (LC); Sequence 4: LC, LE and LP. During the exercise sessions, subjects were verbally encouraged to perform all series until concentric failure, and the same definitions of the movement patterns used during the 10MR test were used to define the conclusion of a repetition.

\section{Statistical treatment}

All data are presented as mean \pm standard deviation (SD). Statistical analysis was initially performed using the Shapiro-Wilk normality test and homoscedasticity test (Bartlett's criteria). All variables have normal distribution and homoscedasticity. The intraclass correlation coefficient $(I C C=(M S b-M S w) /[M S b+(k-1) M S w)$ was calculated to 
verify the reproducibility of the $10 \mathrm{MR}$ test and retest in all exercises. ANOVA was used for repeated measures to compare the averages of the sum of repetitions on the three sets in each exercise and, when needed, a Bonferroni post hoc was used to identify significant differences. In all cases was adopted $p \leq 0.05$ as level of statistical significance. A paired $t$ test was used to compare the results of the total volume of repetitions. The SPSS software version 20.0 was used for statistical analyzes.

\section{RESULTS}

The ICC obtained in $10 \mathrm{MR}$ test and retest were 0.97; 0.96; 0.92; 0.91 and 0.98 in BP, PD, SP, BC and TR exercises, respectively, and 0.99; 0.93; 0.91 in LP, LE and LC exercises, respectively. The sum of the number of maximum repetitions completed in three series for each experimental protocol are shown in Figures 1 and 2.

Significant differences were observed $(p<0.05)$ in the total number of repetitions for BP, SP, BC and TR exercises in the sequence of the upper limbs. For lower limbs, LP and LE exercises (tables 1 and 2 ).

The sequences showed no significant difference in the total volume of repetitions.

\section{DISCUSSION}

The aim of this study was to determine whether the order of the exercises in RT sessions involving lower and upper limbs would affect the total number of repetitions performed in each exercise. The results suggest that regardless of involve upper or lower limbs, the exercises placed at the beginning of the training sessions had higher numbers of repetitions than when performed at the end of the session. There were significant differences in the total number of repetitions in all exercises of the sequence of the upper limbs, with the exception of PD exercise, whereas in the sequence of the lower limbs only LC exercise showed no significant differences between the sequences.

The results found in this study are in agreement with the findings of previous studies. ${ }^{(7-18)}$ Sforzo and Touey ${ }^{(7)}$ examined the influence of exercise order on muscle performance during a TR session. Participated in this study 17 males. Exercises of $U L$ and $L L$ were realized. Regardless of the exercises being in small groups (SG) or large groups (LG) they showed a higher number of repetitions when positioned at the beginning of the training.

Simão et al. ${ }^{(10)}$ examined the influence of exercise order in RT on the number of repetitions and subjective perceived of exertion (SPE). The study included 23 trained female. Six exercises for UL and LL were used, with three series until concentric failure. All the exercises showed significant reductions in the number of repetitions performance, being higher in the first series. In all the exercises significant reductions in the number of repetitions were observed on a

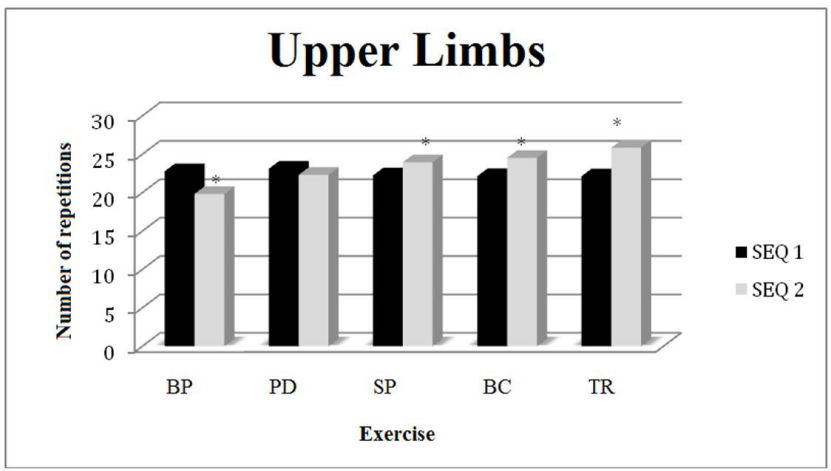

Figure 1. Sum of the number of repetitions in each exercise by sequence. * Significant difference in the number of repetitions between Sequence 1 (SEQ1) and Sequence 2 (SEQ2) BP= bench press, $P D=$ front pull-down, $\mathrm{SP}=$ machine shoulder press, $\mathrm{BC}=$ biceps curl, $\mathrm{TR}=$ triceps.

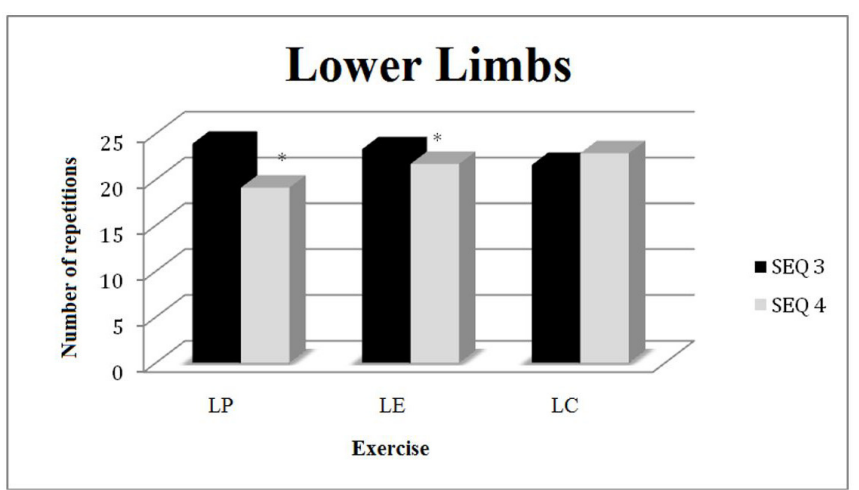

Figure 2. Sum of the number of repetitions in each exercise by sequence. * Significant difference in the number of repetitions between Sequence 3 (SEQ3) and Sequence (SEQ4) LP= leg press, LE= leg extension, LC= leg curl.

Table 1. Average of repetitions per exercise in UL (Mean \pm SD).

\begin{tabular}{lll}
\hline \multicolumn{1}{c}{ Exercise } & Sequence 1 & Sequence 2 \\
\hline Bench press & $8.16 \pm 1.90$ & $7.11 \pm 2.21^{*}$ \\
Front Pull-down & $8.31 \pm 1.60$ & $8.03 \pm 1.92$ \\
Machine shoulder press & $7.97 \pm 1.93$ & $8.61 \pm 1.27^{*}$ \\
Biceps curl & $7.94 \pm 1.91$ & $8.81 \pm 1.21^{*}$ \\
Triceps & $7.94 \pm 1.90$ & $9.31 \pm 0.67^{*}$ \\
\hline
\end{tabular}

* Significant difference in the mean of the total of repetitions in the first sequence versus the second sequence of the same exercise.

Table 2. Number of repetitions per exercise in LL (Mean \pm DP).

\begin{tabular}{lll}
\hline \multicolumn{1}{c}{ Exercise } & Sequence 3 & Sequence 4 \\
\hline Leg press & $8.58 \pm 1.30$ & $6.83 \pm 2.31^{*}$ \\
Leg extension & $8.36 \pm 1.36$ & $7.78 \pm 1.91$ \\
Leg curl & $7.75 \pm 1.86$ & $8.19 \pm 1.47$ \\
\hline
\end{tabular}

* Significant difference in the number of repetitions in the first series versus the second series of the same exercise. 
larger scale when the exercises were positioned at the end of training.

Bellezza et al. ${ }^{(17)}$ examined the influence of the exercise order in RT on performance indicators, blood lactate, percentage and affective responses. The study included 29 subjects ( 18 male and 11 female). In this study the training was composed of UL and LL exercises, with two series until concentric failure. The exercises positioned at the beginning of the series showed significant differences, regardless of gender.

Comparing the results found in the above articles with the current study, despite the exercise sequences were merged with UL and LL and different from the current study, the results are similar to the exercises that initiate the sequences.

Figueiredo et al. ${ }^{(18)}$ examined the influence of the exercise order in RT on performance indicators in local muscular endurance and subjective perceived of exertion. The study included 19 trained female. Five exercises for the upper limbs were used with four series until concentric failure. The number of repetitions per set of initial exercises of each sequence was performed at the end of each sequence when showed significant reductions. This data indicates that in trained women, local muscular endurance is affected by the sequence of exercises and when they are performed at the end of the sequence show decrease in the exercise performance as a result of fatigue. According to Figuereido et al., ${ }^{(18)}$ the exercises performed at the beginning of the sequence for UL showed a significant change in the number of repetitions.

The studies described so far have been conducted with trained adults (men and women) and even then similarities with the current study were found.

The present study shows change in the average of total volume of repetitions to UL exercises The present study shows change in the average total volume of repetitions to the exercises for arms positioned at the end of the training sequence corroborating with the findings of Simão et al. (11) which stated that, regardless of the amount of muscular mass involved, were observed change in the number of repetitions for SS and XL. The same study suggests that considering acute responses and chronic adaptations, the exercises should be prioritized and first performed in the session because of the needs of specific individual improvements and the training objective.

Contradicting Faigenbaum et al. ${ }^{(1)}$, which in positioning regarding the resistance training in youth suggested that young people should start training sequences from the largest to the smallest grouping or from the multi-joint exercises to the single joint in order to obtain better results, the current study for teenagers and studies performed for adults with manipulation of the order, suggest that the prioritized groups exercises should start the sequence of training. In addition, efficient training methods are usually recommended to increase the volume of training and reduce the duration of sessions. ${ }^{(19,20,21)}$ Besides, this was the first study of teenagers manipulating the exercise order in a training session, however the results corroborate with studies conducted with adults (men and women) to UL and LL.

\section{CONCLUSION}

Based on the important findings, we conclude that the performance of the number of repetitions for TR sessions conducted with $10 \mathrm{MR}$ intensity in teenagers was influenced by different orderings of the exercises in both upper limbs and lower limbs. The present study suggests that the performed exercises at the beginning of the sequences show higher numbers of repetitions when exercises are performed until the concentric failure with intensities of $10 \mathrm{MR}$ by teenagers. This finding applies to both exercises involving large and small muscle groups. As shown, the maintainability of repetitions of exercises positioned at the end of the session is committed. Therefore, exercises that are considered more important based on individual needs should be performed at the beginning of the sequences to avoid the decrease in the number of repetitions induced by the fatigue.

\section{AUTHOR'S CONTRIBUTIONS}

MPN, EM, HM, RS, MFM- Article Design; EM, MPN, BFS, HM, MFM - Data collection and search for articles; RS, HM e BFS - Guidance and development

\section{CONFLICTS OF INTEREST}

The authors declare that they have no conflicts of interest in the research.

\section{AUTHOR DETAILS}

${ }^{1}$ Escola de Educação Física e Desportos, Universidade Federal do Rio de Janeiro - UFRJ, Rio de Janeiro (RJ), Brasil.

${ }^{2}$ Mestre em Educação Física, Escola de Educação Física e Desportos, Universidade Federal do Rio de Janeiro- UFRJ, Rio de Janeiro (RJ), Brasil.

${ }^{4}$ Doutor em Fisiopatologia Clínica, Universidade do Estado do Rio de Janeiro - UFRJ, Rio de Janeiro (RJ), Brasil.

${ }^{5}$ Professor Doutor Adjunto, Escola de Educação Física e Desportos, Universidade Federal do Rio de Janeiro- UFRJ, Rio de Janeiro (RJ), Brasil.

${ }^{6}$ Professor PhD Adjunto, Escola de Educação Física e Desportos, Universidade Federal do Rio de Janeiro- UFRJ, Rio de Janeiro (RJ), Brasil.

\section{REFERENCES}

1. Faigenbaum AD, Kraemer WJ, Blimkie CJ, Jeffreys I, Micheli LJ, Nitka et al. Youth resistance training: updated position statement paper from the national strength and conditioning association. J Strength Cond Res. 2009(23):60-79.

2. Ramsay J, Blimkie C, Smith K, Garner S, Macdougall J. Strength training effects in prepubescent boys. Med Sci Sports Exerc. 1990(22):605-14.

3. Morris F, Naughton G, Gibbs J, Carlson J, Wark J. Prospective ten-month exercise intervention in premenarcheal girls: Positive effects on bone and lean mass. J Bone Miner Res. 1997;12:1453-62.

4. American Academy of Pediatrics. Strength Training by Children and Adolescents. Pediatrics. 2008;121:834-40.

5. Granacher U, Goesele A, Roggo K, Wischer T, Fischer S, Zuerny C, et al. Effects and mechanisms of strength training in children. Int J Sports Med. 2011;32:357-64.

6. American College of Sports Medicine. Position stand: Progression models in resistance training for healthy adults. Med Sci Sports Exerc. 2009;41:687-8. 
7. Sforzo GA, Touey PR. Manipulating exercise order affects muscular performance during a resistance exercise training session. J Strength Cond Res. 1996;10:20-4.

8. Simão R, Farinatti PT, Polito MD, Maior AS, Fleck SJ. Influence of exercise order on the number of repetitions performed and perceived exertion during resistance exercises. J Strength Cond Res. 2005;19:152-6.

9. Spreuwenberg LP, Kraemer WJ, Spiering BA, Volek JS, Hatfield DL, Silvestre $\mathrm{R}$, et al. Influence of exercise order in a resistance-training exercise session. J Strength Cond Res. 2006;20:141-4.

10. Simão R, Farinatti PT, Polito MD, Viveiros L, Fleck SJ. Influence of exercise order on the number of repetitions performed and perceived exertion during resistance exercise in women. J Strength Cond Res. 2007;21:23-8.

11. Simão R, Salles BF, Figueiredo T, Dias I, Willardson JM. A review exercise order in resistance training. Sports Med. 2012;42:251-65.

12. Simão R, Figueiredo T, Leite RD, Jansen A, Willardson JM. Influence of exercise order on repetition performance during low-intensity resistance exercise. Res Sports Med. 2012;20(3-4);263-73.

13. Chaves CP, Simão R, Miranda H, Ribeiro J, Soares J, Salles B, et al. Influence of exercise order on muscle damage during moderate-intensity resistance exercise and recovery. Res Sports Med. 2013;21(2):176-86.

14. Miranda H, Figueiredo T, Rodrigues B, Paz GA, Simão R. Influence of exercise order on repetition performance among all possible combinations on resistance training. Res Sports Med. 2013;21(4):355-66.
15. Romano N, Alves J, Fernandes HM, Saavedra F, Paz G, Miranda H, et al. Effects of resistance exercise order on the number of repetitions performed to failure and perceived exertion in untrained young males. $J$ Hum Kinet. 2013;39:177-83.

16. Duke PM, Litt IF, Gross RT. Adolescents self-assessment of sexual maturation. Pediatrics. 1980;66:918-20.

17. Bellezza PA, Hall EE, Miller PC, Bixby WR. The influence of exercise order on blood lactate, perceptual, and affective responses. J Strength Cond Res. 2009;23:203-8.

18. Figueiredo T, Rhea MR, Bunker D, Dias I, Salles BF, Fleck S, et al. The influence of order on local muscular endurance during resistance training in women. Hum Movim. 2011;12:237-41.

19. Paz GA, Willardson JM, Simão R, Miranda R. Effect of different antagonist protocols on repetition performance and muscle activation. Med Sportiva. 2013;17(3):106-12.

20. Paz GA, Robbins DW, Oliveira CG, Bottaro M, Miranda H. Volume load and neuromuscular fatigue during an acute bout of agonist-antagonist paired-set versus traditional-set training. J Strength Cond Res. 2015;in press.

21. Paz GA, Maia MF, Lima VP, Oliveira CG, Bezerra E, Simão R, et al. Maximal exercise performance and electromyography responses after antagonist neuromuscular proprioceptive facilitation: A pilot study. JEPonline. 2012;15(6):60-7. 